\title{
First evaluation of QuantiFERON-TB Gold Plus performance in contact screening
}

\author{
Lucia Barcellini ${ }^{1}$, Emanuele Borroni ${ }^{1}$, James Brown ${ }^{2}$, Enrico Brunetti ${ }^{3}$, \\ Daniela Campisi ${ }^{4}$, Paola F. Castellotti ${ }^{4}$, Luigi R. Codecasa ${ }^{4}$, Federica Cugnata ${ }^{5}$, \\ Clelia Di Serio ${ }^{5}$, Maurizio Ferrarese ${ }^{4}$, Delia Goletti ${ }^{6}$, Marc Lipman $^{2}$, \\ Paola M.V. Rancoita ${ }^{5}$, Giulia Russo ${ }^{1}$, Marina Tadolini ${ }^{7}$, Elisa Vanino ${ }^{7}$ and \\ Daniela M. Cirillo ${ }^{1}$
}

Affiliations: ${ }^{1}$ Emerging Bacterial Pathogens Unit, Division of Immunology and Infectious Diseases IRCCS, San Raffaele Scientific Institute, Milan, Italy. ${ }^{2}$ Royal Free London NHS Trust and UCL Respiratory, Division of Medicine, University College London, London, UK. ${ }^{3}$ Dept of Infectious Diseases, San Matteo Hospital Foundation, University of Pavia, Pavia, Italy. ${ }^{4}$ Regional TB Reference Centre and Laboratory, Villa Marelli Institute/Niguarda Ca' Granda Hospital, Milan, Italy. ${ }^{5}$ University Centre of Statistics for Biomedical Sciences (CUSSB), Vita-Salute San Raffaele University, Milan, Italy. ${ }^{6}$ Translational Research Unit, Epidemiology Dept, National Institute for Infectious Diseases, Rome, Italy. ${ }^{7}$ Infectious Diseases Unit, Dept of Medical and Surgical Sciences, Alma Mater Studiorum University of Bologna, Bologna, Italy.

Correspondence: Daniela M. Cirillo, Emerging Bacterial Pathogens Unit, San Raffaele Scientific Institute, Via Olgettina 58, 20132 Milan, Italy. E-mail: cirillo.danielaahsr.it

ABSTRACT Identifying latently infected individuals is crucial for the elimination of tuberculosis (TB). We evaluated for the first time the performance of a new type of interferon- $\gamma$ release assay, QuantiFERONTB Plus (QFT-Plus), which includes an additional antigen tube (TB2), stimulating both $\mathrm{CD} 4^{+}$and $\mathrm{CD} 8^{+} \mathrm{T}$ cells in contacts of TB patients.

Contacts were screened for latent TB infection by tuberculin skin test, QFT-Plus and QuantiFERON-TB Gold in Tube (QFT-GIT).

In 119 TB contacts, the overall agreement between QFT-Plus and QFT-GIT was high, with a Cohen's $\kappa$ of 0.8. Discordant results were found in 12 subjects with negative QFT-GIT and positive QFT-Plus results. In analyses of markers of $\mathrm{TB}$ exposure and test results, the average time spent with the index case was the strongest risk factor for positivity in each of these tests. The difference in interferon- $\gamma$ production between the two antigen tubes (TB2-TB1) was used as an estimate of $\mathrm{CD}^{+}$stimulation provided by the TB2. TB2-TB1 values $>0.6 \mathrm{IU} \cdot \mathrm{mL}^{-1}$ were significantly associated with proximity to the index case and European origin.

QFT-Plus has a stronger association with surrogate measures of TB exposure than QFT-GIT in adults screened for latent TB infection. Interferon- $\gamma$ response in the new antigen tube used an indirect estimate of specific $\mathrm{CD}^{+}$response correlates with increased Mycobacterium tuberculosis exposure, suggesting a possible role in identifying individuals with recent infection.

@ERSpublications

QuantiFERON-TB Plus improved the diagnostic accuracy for latent TB infection in the setting of contact screening http://ow.ly/2Az0300SDg3 


\section{Introduction}

Despite the progress recently made in tuberculosis (TB) control at a global level, the decline in TB incidence is much slower than that needed to achieve TB elimination by 2050 [1]. Identifying and treating symptom-free people who are truly latently infected with Mycobacterium tuberculosis is key to achieving this $[2,3]$. The current global burden of latent infection is uncertain, although it has been suggested that one-third of the world's population may be latently infected with M. tuberculosis [4].

Although they show no sign of disease, individuals with latent TB infection (LTBI) are at risk of reactivation and up to $10 \%$ of them may develop active disease in their lifetime [5]. This risk is highest in the first 2 years following infection. Preventive treatment of recently infected individuals reduces this [6]. However, isoniazid preventive therapy is not optimal for a large-scale implementation programme, and the current LTBI diagnostic tests (tuberculin skin test (TST) and interferon (IFN)- $\gamma$ release assays (IGRAs)) have significant limitations. TST may produce a false-positive result due to sensitisation by environmental mycobacteria and bacille Calmette-Guerin (BCG) vaccination [7]. In recent decades IGRAs measuring the IFN- $\gamma$ concentration after in vitro whole-blood stimulation with peptides from the RD-1 region of the M. tuberculosis genome have been developed to improve the specificity of the diagnosis [8]. IGRAs are a useful indicator of M. tuberculosis exposure, as their specificity is very high (97\%) [9]. However, like TST, they lose sensitivity in immune-compromised individuals and children [10,11], they identify both recent and past infection and they are poor at predicting LTBI subjects who are at greater risk of developing disease (positive predictive value IGRA 2.7\% and TST 1.5\%) [12]. As a result, when currently available diagnostic tests are used to guide the administration of preventive therapy, the number needed to treat to prevent one case of TB is too high to allow a large-scale preventive programme. Different approaches have been described in the literature to help discriminate those at greater risk of active TB development. The use of IFN- $\gamma$ response to the latency antigen heparin-binding-haemoagglutinin $[13,14]$, immunoprofiling $[15,16]$, gene expression pattern (i.e. interleukin-13 and autoimmune regulator (AIRE)) $[17,18]$ and proportion of peripheral blood monocytes [19] have been studied as possible biomarkers for incipient TB. However, all of these approaches are still confined to research fields and currently have minimal impact on patient management.

QuantiFERON-TB Plus (QFT-Plus; Qiagen, Hilden, Germany) is a new generation of QTF-Gold in Tube (QFT-GIT) [5] that includes an additional antigen tube (TB2). The TB1 tube contains ESAT-6- and CFP-10-derived peptides (TB-7.7, present in QFT-GIT, has been removed), designed to elicit cell-mediated immune responses from $\mathrm{CD} 4^{+}$T-helper lymphocytes. TB2 contains new peptides able to stimulate IFN- $\gamma$ production by both $\mathrm{CD} 4^{+}$and $\mathrm{CD} 8^{+} \mathrm{T}$-cells [20].

Evidence supports the important contribution of $\mathrm{CD}^{+}$T-cells in host defence against $M$. tuberculosis by both cytokine secretion and cytotoxic activity [21]. Firstly, a positive correlation between specific $\mathrm{CD}^{+}$ T-cells and increased mycobacterial load has been found in peripheral blood ex vivo [22]. DAY et al. reported that $>60 \%$ of individuals with smear-positive $\mathrm{TB}$ had detectable $\mathrm{CD} 8^{+} \mathrm{T}$-cell response, compared with $38 \%$ and $20 \%$ of smear-negative and LTBI individuals, respectively. Consistent with this paradigm, a higher prevalence of $M$. tuberculosis-specific $\mathrm{CD}^{+}$T-cells have been reported in smear-positive versus smear-negative patients and in pulmonary TB compared with extrapulmonary TB [23]. In addition, a positive correlation between the $\mathrm{CD}^{+} \mathrm{T}$-cell response against $\mathrm{TB}$ antigens and recent exposure to M. tuberculosis have been found. Recent contacts of active TB patients, independent of their response to QTF, have a greater $\mathrm{CD}^{+} \mathrm{T}$-cell response compared to other study groups (active TB patients, healthcare workers and BCG-vaccinated healthy controls) [24]. This is in agreement with findings observed in a cattle model where a $\mathrm{CD}^{+} \mathrm{T}$-cell response is present at the onset of infection [25].

The IFN- $\gamma$ release assays currently in use primarily elicit a $\mathrm{CD}^{+}$response, but emerging data provide a good rationale for measuring specific $\mathrm{CD}^{+} \mathrm{T}$-cell responses, and in particular to further investigate the association between $\mathrm{CD} 8^{+} \mathrm{T}$-cells and risk of disease progression.

In the present study we evaluate the performance characteristics of the new QFT-Plus assay in TST-positive contacts with recent exposure to people with confirmed active TB, assessing the use of QFT-Plus head-to-head with the previous QFT-GIT. In addition, we investigate for the first time the significance and the possible use of the $\mathrm{CD} 8^{+}$IFN- $\gamma$ response provided by the second, newly added antigen tube.

\section{Materials and methods}

\section{Study setting and participants}

We conducted a cross-sectional study at Villa Marelli-Niguarda Hospital (Milan, Italy). TB incidence in Milan is 16.6 new cases per 100000 person-years (in 2011) [26], three times higher than the Italian national average. From November 2014 to June 2015 we prospectively recruited TST-positive (TST $\geqslant 5 \mathrm{~mm}$ ) contacts of notified active TB cases sent by the local public health services to be screened for LTBI. 
Contacts were excluded if aged $<18$ years, a previous positive TST was documented, preventive TB treatment was prescribed or past TB history was reported. Informed written consent was obtained from each study subject. Contacts reporting mild or severe immunosuppression (diabetes mellitus, chronic kidney disease, HIV, malignancy or immunosuppressive medications) were included.

The study was approved by the ethics committee of the San Raffaele Scientific Institute (Milan, Italy).

Contact screening strategy was based on the National Institute of Health and Care Excellence TB guidelines 2011 [27] and Italian guidelines which recommend retesting those with positive TST results using an IGRA as a confirmatory test. At the contact's first visit health status was established by clinical examination and chest radiography. Further information on the country of birth, immigration status, nature of the contact to the source case, BCG vaccination status (if details were unclear, inspection of BCG vaccination scar was performed by trained healthcare assistants) and clinical history were obtained through personal interviews. When clinical suspicion persisted, chest computed tomography scans and sputum sample analyses were requested.

All patients also underwent testing in line with recommended routine screening as part of contact investigation. Thus, TST-positive contacts who tested negative to a first QFT-GIT analysis were retested using QFT-GIT after 10-12 weeks to exclude delayed conversion.

Blood samples were obtained for QFT-GIT, QFT-Plus and HIV testing from all subjects providing informed consent. QFT-GIT currently in use in clinical practice was performed at Niguarda microbiology service (Milan, Italy), while QFT-Plus testing was performed in the emerging bacterial pathogen laboratory at San Raffaele Hospital. The QFT-Plus and QFT-GIT tests were performed according to the manufacturer's instructions. Peripheral blood samples for the two tests were obtained simultaneously directly into the QFT tubes and processed within $4 \mathrm{~h}$. Test interpretation for both QFT-Plus and QFT-GIT was performed according to the manufacturer's instruction manual. QFT-GIT results were recorded positive if the antigen response was $>0.35 \mathrm{IU} \cdot \mathrm{mL}^{-1}$ above the negative control response. Positivity (antigen response $>0.35 \mathrm{IU} \cdot \mathrm{mL}^{-1}$ above the negative control response) of a single antigen tube (either TB1 or TB2) was sufficient to score the QFT-Plus test as positive.

\section{Ascertainment of exposure}

We assessed different factors as surrogate markers of $M$. tuberculosis exposure. The aggregate exposure time of contacts prior to the diagnosis of their respective source case was established by recording the extent of the contact during a typical week. TB contacts were categorised according to proximity to the index case [28]: we considered them to be "high proximity" if contacts and case patients were routinely sharing the same bedroom and lower proximity if contacts and case patients were sleeping in a different bedroom in the same house or in a different house. Sputum smear positivity of the index case was also assessed as TB case-related risk factor.

\section{Statistical analysis}

The agreement between QFT-GIT and QFT-Plus was evaluated by computing the overall percentage of concordant results and Cohen's $\kappa$ coefficient with $95 \%$ confidence intervals.

Univariate logistic regression and backward stepwise multivariate logistic regression models were used to identify factors associated with positive test results. The variables considered in the analyses were sex, whether the country of birth was a TB-endemic area and whether it was in Europe, BCG vaccination, immunocompromised status, smear status of the index case, average time spent per week with the index case and place of sleeping with respect to the index case. The same analysis was performed for the variable denoting whether the differences between QFT-Plus TB2 and QFT-Plus TB1 was greater than the cut-off $0.6 \mathrm{IU} \cdot \mathrm{mL}^{-1}$ (as described in the results section). The level of significance considered was $5 \%$. All statistical analyses were performed using R statistical software (version 3.2.3; www.r-project.org).

\section{Results}

119 M. tuberculosis-exposed individuals with positive TST $(\geqslant 5 \mathrm{~mm})$ were investigated. Of these, 39 were contacts of smear-negative culture-positive $\mathrm{TB}$ cases, and 69 were contacts of smear-positive culture-positive index cases. Participants had a median (interquartile range) age of 38 (30-79) years, more than half $(\mathrm{n}=61,51.26 \%)$ were non-European-born, 82 (78.85\%) were BCG vaccinated and $11(9.24 \%)$ were immunocompromised subjects. Demographic characteristics of the cohort are shown in table 1. 


$\begin{array}{lc}\text { TABLE } 1 \text { Demographic characteristics } & 119 \\ \text { Sex } & 63(52.9) \\ \text { Male } & 56(47.1) \\ \text { Female } & 119 \\ \text { Estimated incidence of TB in country of birth" } & 48(40.4) \\ 0-50 \text { per } 100000 \text { person-years } & 71(59.7) \\ >50 \text { per } 100000 \text { person-years } & 119 \\ \text { Country of birth } & 58(48.7) \\ \text { European } & 61(51.3) \\ \text { Non-European } & 104 \\ \text { BCG vaccination } & 22(21.1) \\ \text { No } & 82(78.8) \\ \text { Yes } & 108 \\ \text { Smear status of index case } & 39(36.1) \\ \text { Negative } & 69(63.9) \\ \text { Positive } & 108 \\ \text { Time spent with the index case h per day } & 27(25) \\ 1-4 & 25(23.1) \\ 5-8 & 9(8.3) \\ 9-12 & 47(43.5) \\ >12 & \\ \text { Sleeping proximity to the index case } & 61(56.5) \\ \text { Different house } & 19(17.6) \\ \text { Different rooms } & 28(25.9) \\ \text { Same room } & 119 \\ \text { Immunocompromised" } & 108(90.8) \\ \text { No } & 11(9.2) \\ \text { Yes } & \end{array}$

Data are presented as $\mathrm{n}$ or $\mathrm{n}(\%)$. TB: tuberculosis; BCG: bacille Calmette-Guerin. ${ }^{\#}$ : as per World Health Organization report 2014 [29]; ๆ: causes of immunosuppression: diabetes mellitus ( $n=6)$, chronic kidney disease ( $n=0)$, HIV ( $n=2)$, malignancy $(n=2)$ and immunosuppressive medications ( $n=1)$.

were positive to QFT-GIT. The overall agreement between the two IGRAs was high, with a Cohen's $\kappa$ of 0.8 (95\% CI 0.69-0.91). The two tests gave concordant results for 107 (89.9\%) subjects (table 2). Discordant results were found in 12 subjects: they all scored negative to the QFT-GIT test and positive to the QFT-Plus test. Discordant results between the two IGRAs included the four contacts with a single-tube QFT-Plus positivity. Moreover, contacts with IGRA-discordant results had overall low IFN- $\gamma$ responses, but not so low to be considered borderline results (median TB1-nil $0.83 \mathrm{IU} \cdot \mathrm{mL}^{-1}$ and TB2-nil $\left.0.73 \mathrm{IU} \cdot \mathrm{mL}^{-1}\right)$. The characteristics of subjects with discordant results are shown in table 3 . Only one of the 12 contacts with QFT-Plus positive and QFT-GIT negative results had a TST response $<10 \mathrm{~mm}(7 \mathrm{~mm})$. Globally, the median TB1 QFT-Plus antigen IFN- $\gamma$ level (TB1-Nil) was $0.74 \mathrm{IU} \cdot \mathrm{mL}^{-1}$, whereas the median TB2 QFT-Plus antigen IFN- $\gamma$ level (TB2-Nil) was $0.67 \mathrm{IU} \cdot \mathrm{mL}^{-1}$, as reported in table 2.

As per the Italian guidelines, contacts of TB cases with initial positive TST results who tested negative to a first QFT-GIT analysis were retested with QFT-GIT at 10-12 weeks. At the post-exposure follow-up, two contacts converted to QFT-GIT positive results. They were both part of the 12 contacts who initially showed QFT-Plus positive/QFT-GIT negative discordant results (table 2). One of them had a strong QFT-GIT positivity $\left(>10 \mathrm{IU} \cdot \mathrm{mL}^{-1}\right)$ at 10 weeks post-exposure follow-up, while the second case reported a

\section{TABLE 2 Test results}

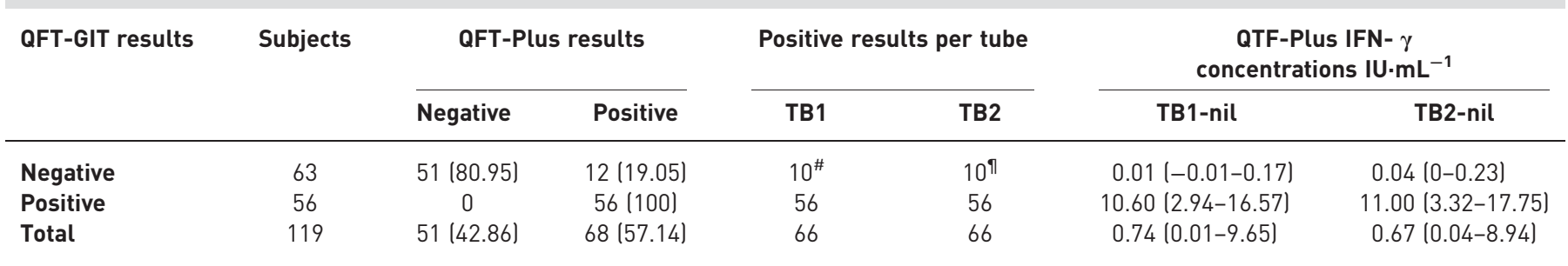

Data are presented as $\mathrm{n}, \mathrm{n}(\%)$ or median (interquartile range). ${ }^{*}$ : two were positive to TB1 only; ${ }^{\text {ๆ: }}$ two were positive to TB2 only. QFT-GIT: QuantiFERON-TB Gold in Tube; QFT-Plus: QuantiFERON-TB Plus; IFN: interferon. 
TABLE 3 QuantiFERON-TB Plus (QFT-Plus) and QuantiFERON-TB Gold in Tube (QFT-GIT) discordant results

\begin{tabular}{|c|c|c|c|c|c|c|c|c|}
\hline Sample & BCG scar & $\begin{array}{l}\text { TST diameter } \\
\text { of induration } \mathrm{mm}\end{array}$ & QFT-GIT & $\begin{array}{c}\text { QFT-Plus } \\
\text { TB1 }{ }^{\#} \text { IU.mL } \mathrm{mL}^{-1}\end{array}$ & $\begin{array}{c}\text { QFT-Plus } \\
\text { TB2 }^{\text {TI IU.mL }} \mathrm{mL}^{-1}\end{array}$ & $\begin{array}{l}\text { Index case } \\
\text { smear status }\end{array}$ & $\begin{array}{l}\text { Relationship } \\
\text { to index case }\end{array}$ & Immunosuppression \\
\hline C1 & Yes & 20 & Negative & 1.83 & 0.51 & Positive & $\begin{array}{l}\text { Household, primary } \\
\text { caregiver }\end{array}$ & Prednisone treatment \\
\hline C11 & Yes & 7 & Negative $^{+}$ & 0.49 & 0.83 & Positive & Boyfriend & No \\
\hline C15 & No & 21 & Negative $^{+}$ & 0.11 & 0.48 & Positive & $\begin{array}{c}\text { Employer (index case: } \\
\text { housemaid) }\end{array}$ & No \\
\hline C17 & Yes & 10 & Negative & 0.38 & 0.41 & Negative & Household, sister & No \\
\hline C53 & Yes & 20 & Negative & 0.3 & 0.58 & Positive & $\begin{array}{c}\text { Colleague, daily journey } \\
\text { to work }\end{array}$ & No \\
\hline C63 & Yes & 16 & Negative & 0.74 & 0.67 & Negative & Household & No \\
\hline $\mathrm{C} 69$ & Yes & 14 & Negative & 0.52 & 0.29 & Positive & Household & No \\
\hline C75 & Yes & 11 & Negative & 0.81 & 0.9 & Positive & Household & No \\
\hline C78 & No & 11 & Negative & 1.88 & 1.93 & Positive & $\begin{array}{l}\text { Colleague (sharing the } \\
\text { same room) }\end{array}$ & No \\
\hline
\end{tabular}

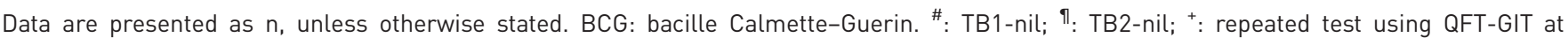
follow-up converted to positive.

QFT-GIT of $0.5 \mathrm{IU} \cdot \mathrm{mL}^{-1}$ after 6 months of isoniazid preventive therapy (decision to treat was based on the strong TST positivity and the proximity of contact with the index case). In both cases the TB2 IFN- $\gamma$ response was greater than that found in TB1.

\section{Independent predictors of QFT-Plus and QFT-GIT positivity}

For both QFT-GIT and QFT-Plus tests, the univariate odds ratios of being positive for different possible surrogate markers of increasing exposure to M. tuberculosis are presented in table 4. Contacts reporting that they had spent $>12 \mathrm{~h}$ per day with the index case were significantly more likely to be both QFT-GIT and QFT-Plus positive, compared to contacts spending 1-4 h per day with the index case. For a subject with an exposure time $>12 \mathrm{~h}$ per day, the odds of a positive test were six times higher by QFT-GIT and 14 times higher by QFT-Plus. Both test results were significantly more likely to be positive in subjects with closer sleeping proximity to the patient (same house versus different house). The odds of being QFT-GIT positive for subjects sleeping in the same house of the index case were approximately four times higher (different rooms 3.79, same room 3.98) than for those sleeping in a different house, whereas their odds of being QFT-Plus positive were approximately six times higher (different rooms 5.78, same room 5.65). The results of the backward stepwise multivariate logistic regression analysis are presented in table 5. Only the variable indicating whether a contact spent on average $>12 \mathrm{~h}$ per day with the index case remained significantly associated with a positive QFT-GIT result (OR 4.63, 95\% CI 2.05-10.47) and a positive QFT-Plus result (OR 6.98, 95\% CI 2.86-17.02).

\section{Predictors for $\mathrm{CD8^{+ }} T$-cell stimulation}

To assess the specific contribution of $\mathrm{CD}^{+} \mathrm{T}$-cells, we subtracted the quantitative value of the first antigen tube expressed in IU.mL $L^{-1}$ (TB1), which stimulates the $\mathrm{CD} 4^{+}$population only, from the value provided by the second antigen tube (TB2), in which a combined $\mathrm{CD} 4^{+}$and $\mathrm{CD} 8^{+}$T-cell stimulation occurred. We used a difference of $0.6 \mathrm{IU} \cdot \mathrm{mL}^{-1}$ to define positive results in order to reduce the bias of the intrinsic variability of the test [30]. 18 out of 119 (15.13\%) contacts had a difference between TB2 and TB1 $>0.6 \mathrm{IU} \cdot \mathrm{mL}^{-1}$. Univariate logistic regression was used to identify factors associated with differences between TB2 and TB1 >0.6 IU.mL ${ }^{-1}$ (table 4). This method identified sleeping in the same room compared to sleeping in different houses (OR 4.34, 95\% CI 1.37-13.81) and European origin (OR 3.24, 95\% CI 1.07-9.75) to be to be significantly positively associated with a greater TB2 response. These associations persisted in the multivariate analysis, shown in table 6.

\section{Discussion}

We provide the first evaluation of the QFT-Plus assay alongside the previous version QFT-GIT in a cohort of TST-positive contacts of active TB cases.

Positive results from QFT-Plus were associated with surrogate markers of increasing recent exposure to M. tuberculosis. Paired comparison between QFT-GIT and QFT-Plus shows an overall good, but not 
TABLE 4 Univariate logistic regressions

\begin{tabular}{|c|c|c|c|c|c|c|}
\hline & \multicolumn{2}{|c|}{ QFT-GIT positive } & \multicolumn{2}{|c|}{ QFT-Plus positive } & \multicolumn{2}{|c|}{ TB2-TB1 >0.6 } \\
\hline & OR $(95 \% \mathrm{CI})$ & p-value & OR $(95 \% \mathrm{CI})$ & p-value & OR $(95 \% \mathrm{CI})$ & p-value \\
\hline Age & $1.02(1.00-1.05)$ & 0.09 & $1.02(1.00-1.05)$ & 0.101 & $1.03(1.00-1.07)$ & 0.053 \\
\hline Male & 1 & & 1 & & 1 & \\
\hline Female & $0.73(0.35-1.5)$ & 0.387 & 0.76 (0.37-1.57) & 0.458 & $1.49(0.55-4.10)$ & 0.435 \\
\hline \multicolumn{7}{|c|}{$\begin{array}{l}\text { Estimated incidence of TB per } \\
100000 \text { person-years in country of birth }\end{array}$} \\
\hline$>50$ & $1.44(0.69-3.01)$ & 0.333 & $1.63(0.78-3.42)$ & 0.197 & $0.82(0.30-2.25)$ & 0.7 \\
\hline \multicolumn{7}{|l|}{ Country of birth } \\
\hline Non-European & 1 & & 1 & & 1 & \\
\hline European & $0.84(0.41-1.73)$ & 0.635 & $0.74(0.36-1.54)$ & 0.428 & 3.24 (1.07-9.75) & 0.037 \\
\hline \multicolumn{7}{|l|}{ BCG vaccination } \\
\hline No & 1 & & 1 & & 1 & \\
\hline \multicolumn{7}{|c|}{ Time spent with the index case $h$ per day } \\
\hline $1-4$ & 1 & & 1 & & 1 & \\
\hline $5-8$ & $1.65(0.48-5.67)$ & 0.429 & $3.23(0.97-10.72)$ & 0.055 & $3.55(0.34-36.53)$ & 0.288 \\
\hline $9-12$ & $2.8(0.57-13.83)$ & 0.206 & $4.37(0.89-21.61)$ & 0.070 & $7.43(0.59-94.26)$ & 0.122 \\
\hline$>12$ & $6.78(2.28-20.16)$ & 0.0006 & $14.78(4.62-47.25)$ & $5.6 e-06$ & $7.03(0.85-58.2)$ & 0.071 \\
\hline \multicolumn{7}{|c|}{ Sleeping proximity to the index case } \\
\hline Different house & 1 & & 1 & & 1 & \\
\hline Different rooms & $3.79(1.29-11.14)$ & 0.015 & $5.78(1.71-19.52)$ & 0.005 & $0.51(0.06-4.52)$ & 0.545 \\
\hline Same room & $3.98(1.55-10.23)$ & 0.004 & $5.65(2.00-15.97)$ & 0.001 & $4.34(1.37-13.81)$ & 0.013 \\
\hline \multicolumn{7}{|c|}{ Immunocompromised } \\
\hline No & 1 & & 1 & & 1 & \\
\hline Yes & $0.62(0.17-2.22)$ & 0.459 & $1.35(0.37-4.88)$ & 0.649 & $0.54(0.06-4.46)$ & 0.563 \\
\hline
\end{tabular}

QFT-GIT: QuantiFERON-TB Gold in Tube; QFT-Plus: QuantiFERON-TB Plus; TB: tuberculosis; BCG: bacille Calmette-Guerin. ${ }^{\#}$ : as per World Health Organization report 2014 [29].

complete, agreement. Furthermore, in the majority of cases, the overall IFN- $\gamma$ response in QFT-Plus positive/QFT-GIT negative contacts was out of the uncertainty zone for test interpretation [30], suggesting that differences between the tests are not due to test variability. Of note, the disagreement between the two tests all goes in the same direction, with a total of 12 TST-positive contacts positive with the new QFT-Plus and negative to QFT-GIT. With no gold standard for LTBI to refer to, it is difficult to assess whether the discordant results found during the contact screening are attributable to the higher sensitivity of the QFT-Plus test. If the TST were taken as the reference test for LTBI, this would mean that the proportion of TST-positive contacts confirmed by the IGRA test is increased by $17 \%$ when using the QFT-Plus compared to QFT-GIT. False positivity with TST is mainly due to sensitisation by BCG vaccination [7]. QFT-Plus specificity in a BCG-vaccinated population has not yet been investigated; however, we found that QFT-Plus is not associated with BCG vaccination both in univariate and multivariate analysis. Moreover, only one of the 12 contacts with QFT-Plus positive and QFT-GIT negative result had a TST response $<10 \mathrm{~mm}$, while another showed an intense TST positivity, which is less likely to be the result of previous vaccination [7].

TABLE 5 Backward stepwise multivariate logistic regressions for predicting QuantiFERON-TB Gold in Tube (QFT-GIT) or QuantiFERON-TB Plus (QFT-Plus) positivity

\begin{tabular}{|c|c|c|c|c|}
\hline & \multicolumn{2}{|c|}{ QFT-GIT positive } & \multicolumn{2}{|c|}{ QFT-Plus positive } \\
\hline & OR $(95 \% \mathrm{CI})$ & p-value & OR $(95 \% \mathrm{CI})$ & $p$-value \\
\hline \multicolumn{5}{|c|}{ Time spent with the index case $h$ per day } \\
\hline $1-12$ & 1 & & 1 & \\
\hline$>12$ & $4.63(2.05-10.47)$ & 0.0002 & $6.98(2.86-17.02)$ & $1.98 \times 10^{-5}$ \\
\hline
\end{tabular}




$\begin{array}{lcc}\text { TABLE } 6 \text { Backward stepwise multivariate logistic regression for predicting TB2-TB1 } & \text { p-value } \\ >0.6 \mathrm{IU} \cdot \mathrm{mL}^{-1} & \text { OR }(95 \% \mathrm{CI}) & \\ & & 0.0453 \\ \begin{array}{l}\text { Country of birth } \\ \text { Non-European } \\ \text { European }\end{array} & 1 & \\ \begin{array}{l}\text { Sleeping proximity to the index case } \\ \text { Different room } \\ \text { Same room }\end{array} & 3.46(1.03-11.69) & 0.0029\end{array}$

Recent findings suggest that the discordance between IGRAs and TST in recently exposed individuals may be related to delayed conversion of IGRAs relative to TST $[31,32]$. In this study we found that most of the discordant cases (QFT-GIT negative/QFT-Plus positive) show intense TST positivity; moreover, we reported a shorter period of conversion for QFT-Plus compared to QFT-GIT at least in two individuals of our cohort. These results suggest that QFT-Plus may be more sensitive in detecting new or recent infection with M. tuberculosis than the QFT-GIT.

Our data demonstrate that risk factors for test positivity were the same for both IGRAs. QFT-Plus showed stronger associations with surrogate measures of recent exposure than QFT-GIT both in univariate and multivariate analysis The average time spent per day with the index case had the strongest association with test positivity.

We investigated for the first time the difference in IFN- $\gamma$ production between the two QFT-Plus tubes and surrogate markers of increasing exposure. TB2-TB1 differential values were used as an indirect estimate of specific $\mathrm{CD}^{+}$stimulation with the newly added antigens. A cut-off value was set at $0.6 \mathrm{IU} \cdot \mathrm{mL}^{-1}$ in order to exclude small variations due to intertest variability [30]. Positive TB2-TB1 differences $\left(>0.6 \mathrm{IU} \cdot \mathrm{mL}^{-1}\right)$ were significantly associated with sleeping proximity to the index case with an odds ratio comparable to the one obtained in the analysis of QFT-GIT and QFT-Plus (sleeping in the same room compared to sleeping in different houses OR 4.34, 95\% CI 1.37-13.81). Moreover, European origin (OR 3.24, 95\% CI 1.07-9.75) was significantly associated with $\mathrm{TB} 2-\mathrm{TB} 1>0.6 \mathrm{IU} \cdot \mathrm{mL}^{-1}$, but was not statistically significant for the QFT-GIT and the QFT-Plus results.

As individuals from European countries have a low risk for $M$. tuberculosis exposure, these findings are consistent with the hypothesis that the difference in response between the TB2 and TB1 tubes could be used as a surrogate marker of recent exposure (linked to the specific index case exposure), and not to previous cumulative $M$. tuberculosis exposure. A recent flow-cytometry study reported a positive correlation between the $\mathrm{CD}^{+}$T-cell response against the QFT-GIT antigens and recent exposure to $M$. tuberculosis in contacts of active TB patients compared to controls (active TB patients, healthcare workers and BCG-vaccinated healthy controls) [24].

Tests currently used for M. tuberculosis infection diagnosis do not reflect $\mathrm{CD} 8^{+} \mathrm{T}$-cell cytokine production [33]; however, results reported in previous flow-cytometry studies and our own findings provide a strong rationale for measurement of $M$. tuberculosis-specific $\mathrm{CD}^{+} \mathrm{T}$-cell response. If validated, this may prove to be a surrogate marker of recent infection, which, having the highest risk of progression to active TB, may enable QFT-Plus to distinguish recent infection from long-lasting reactivity and hence allow better targeted delivery of preventive therapy.

M. tuberculosis-specific $\mathrm{CD}^{+} \mathrm{T}$-cells have been more frequently detected in individuals with active TB when compared with LTBI and correlated with increasing antigenic burden [21-23, 34, 35], suggesting that the presence of $\mathrm{CD}^{+}$T-cells in a small proportion of latently infected individuals may be predictive of $M$. tuberculosis active replication and more likely disease progression [22]. Consistent with these results, in a previous study we found that the difference in responses between the QFT-Plus tubes may positively correlate with increasing antigenic load in active TB patients, as it was significantly more common in smear-positive versus smear-negative active TB patients [36]. In the present study, we observed a greater TB2 antigen response (TB2-TB1 difference $>0.6 \mathrm{IU} \cdot \mathrm{mL}^{-1}$ ) in $18(15.13 \%)$ individuals, all QFT-Plus positive. We speculate that the small subgroup of latently infected contacts with TB2-TB1 difference $>0.6 \mathrm{IU} \cdot \mathrm{mL}^{-1}$ had a higher antigenic burden. However, to date, we do not have the tools to directly assess $M$. tuberculosis antigenic burden, as current LTBI tests rely on the (indirect) measurement of a specific immune response.

Our study has limitations. The foremost of these was the sample size, which was 119 subjects. Moreover, because of the lack of gold-standard tests for LTBI, we were unable to adequately resolve the discordance 
between QFT-GIT and QFT-Plus. In addition, TST-negative contacts were not recruited in our sample and a full evaluation of the test would benefit from their presence. Finally, the positive predictive value of the test and of the new parameter, the difference between the two antigen tubes, needs to be properly assessed in a longitudinal cohort. However, this would require follow-up of a large cohort (as incident TB is an uncommon event) and could only be performed in groups who are not eligible for chemoprophylaxis.

To our knowledge, our study is the first evaluation of the QFT-Plus assay among recent contacts of TB cases. Although limited by the small sample size, our data show that QFT-Plus in contact screening has an improved performance compared to QFT-GIT and suggests a role for the differential value between the two tubes as a proxy for recent infection. Larger prospective studies are needed to assess the positive predictive value of the test and the possible role of the differential value between the two antigen tubes as a marker for recent infection.

In conclusion, the difference between the two antigen tubes, used as an indirect estimate of specific CD8 ${ }^{+}$ activation, is associated with factors indicating increased M. tuberculosis exposure, suggesting that this might identify individuals at greater risk of progression to active TB.

QFT-Plus shows stronger association with surrogate measures of exposure compared to QFT-GIT and therefore seems at least as accurate as QFT-GIT in the setting of contact screening.

\section{Acknowledgements}

The authors thank the study subjects for their generous participation and the healthcare assistants and all the staff of the Regional Tuberculosis Reference Centre, Villa Marelli Niguarda Hospital (Milan, Italy) for their diligent work and valuable support.

\section{References}

1 Getahun H, Matteelli A, Chaisson RE, et al. Latent Mycobacterium tuberculosis infection. N Engl J Med 2015; 372: $2127-2135$.

2 Lönnroth K, Migliori GB, Abubakar I, et al. Towards tuberculosis elimination: an action framework for low-incidence countries. Eur Respir J 2015; 45: 928-952.

3 Diel R, Loddenkemper R, Zellweger J-P, et al. Old ideas to innovate tuberculosis control: preventive treatment to achieve elimination. Eur Respir J 2013; 42: 785-801.

4 Dye $\mathrm{C}$, Scheele S, Dolin P, et al. Global burden of tuberculosis: estimated incidence, prevalence, and mortality by country. JAMA 1999; 282: 677-686.

5 Vynnycky E, Fine PE. The natural history of tuberculosis: the implications of age-dependent risks of disease and the role of reinfection. Epidemiol Infect 1997; 119: 183-201.

6 Bass JB, Farer LS, Hopewell PC, et al. Treatment of tuberculosis and tuberculosis infection in adults and children. American Thoracic Society and the Centers for Disease Control and Prevention. Am J Respir Crit Care Med 1994; 149: 1359-1374.

7 Farhat M, Greenaway C, Pai M, et al. False-positive tuberculin skin tests: what is the absolute effect of BCG and non-tuberculous mycobacteria? Int J Tuberc Lung Dis 2006; 10: 1192-1204.

8 Andersen P, Munk ME, Pollock JM, et al. Specific immune-based diagnosis of tuberculosis. Lancet 2000; 356: 1099-1104.

9 Sester M, Sotgiu G, Lange C, et al. Interferon- $\gamma$ release assays for the diagnosis of active tuberculosis: a systematic review and meta-analysis. Eur Respir J 2011; 37: 100-111.

10 Santin M, Muñoz L, Rigau D. Interferon- $\gamma$ release assays for the diagnosis of tuberculosis and tuberculosis infection in HIV-infected adults: a systematic review and meta-analysis. PLoS One 2012; 7: e32482.

11 Mandalakas AM, Detjen AK, Hesseling AC, et al. Interferon-gamma release assays and childhood tuberculosis: systematic review and meta-analysis. Int J Tuberc Lung Dis 2011; 15: 1018-1032.

12 Rangaka MX, Wilkinson KA, Glynn JR, et al. Predictive value of interferon- $\gamma$ release assays for incident active tuberculosis: a systematic review and meta-analysis. Lancet Infect Dis 2015; 12: 45-55.

13 Corbière V, Pottier G, Bonkain F, et al. Risk stratification of latent tuberculosis defined by combined interferon gamma release assays. PLoS One 2012; 7: e43285.

14 Delogu G, Chiacchio T, Vanini V, et al. Methylated HBHA produced in M. smegmatis discriminates between active and non-active tuberculosis disease among RD1-responders. PLoS One 2011; 6: e18315.

15 Escalante P, Peikert T, Van Keulen VP, et al. Catombinatorial immunoprofiling in latent tuberculosis infection. Toward better risk stratification. Am J Respir Crit Care Med 2015; 192: 605-617.

16 Petruccioli E, Petrone L, Vanini V, et al. Assessment of CD27 expression as a tool for active and latent tuberculosis diagnosis. J Infect 2015; 71: 526-533.

17 Rachow A, Heinrich N, Geldmacher C. Early identification of progressive TB disease using host biomarkers. EBioMedicine 2015; 2: 107-108.

18 Zak DE, Penn-Nicholson A, Scriba TJ, et al. A blood RNA signature for tuberculosis disease risk: a prospective cohort study. Lancet 2016 [In press DOI: 10.1016/S0140-6736(15)01316-1].

19 Rakotosamimanana N, Richard V, Raharimanga V, et al. Biomarkers for risk of developing active tuberculosis in contacts of TB patients: a prospective cohort study. Eur Respir J 2015; 46: 1095-1103.

20 QIAGEN. QuantiFERON-TB Gold Plus ELISA Package Insert. 2014. www.quantiferon.com/irm/content/ package-inserts.aspx Date last accessed: March 13, 2015. Date last updated: 2015.

21 Lewinsohn DA, Heinzel AS, Gardner JM, et al. Mycobacterium tuberculosis-specific CD8+ T cells preferentially recognize heavily infected cells. Am J Respir Crit Care Med 2003; 168: 1346-1352.

22 Day CL, Abrahams DA, Lerumo L, et al. Functional capacity of Mycobacterium tuberculosis-specific $\mathrm{T}$ cell responses in humans is associated with mycobacterial load. J Immunol 2011; 187: 2222-2232. 
23 Rozot V, Vigano S, Mazza-Stalder J, et al. Mycobacterium tuberculosis-specific CD8+ T cells are functionally and phenotypically different between latent infection and active disease. Eur J Immunol 2013; 43: 1568-1577.

24 Nikolova M, Markova R, Drenska R, et al. Antigen-specific CD4- and CD8-positive signatures in different phases of Mycobacterium tuberculosis infection. Diagn Microbiol Infect Dis 2013; 75: 277-281.

25 Ryan AA, Nambiar JK, Wozniak TM, et al. Antigen load governs the differential priming of CD8 T cells in response to the bacille Calmette Guérin vaccine or Mycobacterium tuberculosis infection. J Immunol 2009; 182: 7172-7177.

26 Faccini M, Cantoni S, Ciconali G, et al. Tuberculosis-related stigma leading to an incomplete contact investigation in a low-incidence country. Epidemiol Infect 2015; 143: 2841-2848.

27 National Institute for Health and Care Excellence (NICE). Tuberculosis. Clinical Diagnosis and Management of Tuberculosis, and Measures for its Prevention and Control. London, NICE, 2011.

28 Hill PC, Brookes RH, Fox A, et al. Large-scale evaluation of enzyme-linked immunospot assay and skin test for diagnosis of Mycobacterium tuberculosis infection against a gradient of exposure in The Gambia. Clin Infect Dis 2004; 38: 966-973.

29 World Health Organization (WHO). Global Tuberculosis Control. WHO Report 2014. Geneva, WHO, 2014.

30 Metcalfe JZ, Cattamanchi A, McCulloch CE, et al. Test variability of the QuantiFERON-TB gold in-tube assay in clinical practice. Am J Respir Crit Care Med 2013; 187: 206-211.

31 Ribeiro-Rodrigues R, Kim S, Coelho da Silva FD, et al. Discordance of tuberculin skin test and interferon gamma release assay in recently exposed household contacts of pulmonary TB cases in Brazil. PLoS One 2014; 9: e96564.

32 Lee SW, Oh DK, Lee SH, et al. Time interval to conversion of interferon- $\gamma$ release assay after exposure to tuberculosis. Eur Respir J 2011; 37: 1447-1452.

33 Pai M, Dheda K, Cunningham J, et al. T-cell assays for the diagnosis of latent tuberculosis infection: moving the research agenda forward. Lancet Infect Dis 2007; 7: 428-438.

34 Chiacchio T, Petruccioli E, Vanini V, et al. Polyfunctional T-cells and effector memory phenotype are associated with active TB in HIV-infected patients. J Infect 2014; 69: 533-545.

35 Lancioni C, Nyendak M, Kiguli S, et al. CD8+ T cells provide an immunologic signature of tuberculosis in young children. Am J Respir Crit Care Med 2012; 185: 206-212.

36 Barcellini L, Borroni E, Brown J, et al. First independent evaluation of QuantiFERON-TB Plus performance. Eur Respir J 2016; 47: 1587-1590. 